\title{
Repeating Firing Fields of CA1 Neurons Shift Forward in Response to Increasing Angular Velocity
}

\author{
Stephen L. Cowen ${ }^{1}$ and Douglas A. Nitz ${ }^{2}$ \\ ${ }^{1}$ The University of Arizona, Tucson, Arizona 85724-5115, and ${ }^{2}$ The University of California, San Diego, La Jolla, California 92093
}

\begin{abstract}
Self-motion information influences spatially-specific firing patterns exhibited by hippocampal neurons. Moreover, these firing patterns can repeat across similar subsegments of an environment, provided that there is similarity of path shape and head orientations across subsegments. The influence of self-motion variables on repeating fields remains to be determined. To investigate the role of path shape and angular rotation on hippocampal activity, we recorded the activity of CA1 neurons from rats trained to run on spiral-shaped tracks. During inbound traversals of circular-spiral tracks, angular velocity increases continuously. Under this condition, most neurons (74\%) exhibited repeating fields across at least three adjacent loops. Of these neurons, $86 \%$ exhibited forward shifts in the angles of field centers relative to centers on preceding loops. Shifts were absent on squared-spiral tracks, minimal and less reliable on concentric-circle tracks, and absent on outward-bound runs on circular-spiral tracks. However, outward-bound runs on the circular-spiral track in the dark were associated with backward shifts. Together, the most parsimonious interpretation of the results is that continuous increases or decreases in angular velocity are particularly effective at shifting the center of mass of repeating fields, although it is also possible that a nonlinear integration of step counts contributes to the shift. Furthermore, the unexpected absence of field shifts during outward journeys in light (but not darkness) suggests visual cues around the goal location anchored the map of space to an allocentric reference frame.
\end{abstract}

Key words: angular acceleration; hippocampus; memory; navigation; place fields

\section{Introduction}

A defining feature of the firing activity of principal cells within the dorsal hippocampus is their degree of selectivity for specific locations within an environment (O'Keefe and Dostrovsky, 1971; McNaughton et al., 1983b; Muller et al., 1987). This sensitivity, combined with the observation that damage to the hippocampus results in severe impairments in spatial navigation, suggests that networks of hippocampal neurons form the basis of a cognitive map for space (O'Keefe and Nadel, 1978; Moser et al., 2008). Recent observations complicate this picture. Under certain conditions, hippocampal neurons fire across multiple regions of an environment if those regions share common and repeating sensory features and/or share common action sequences. For example, firing fields can repeat within visually similar environments (Skaggs and McNaughton, 1998) or along traversals of common repeating elements of $\mathrm{W}$-shaped, zigzag (hairpin), circular, and spiral-shaped tracks (Derdikman et al., 2009; Singer et al., 2010; Hayman et al., 2011; Nitz, 2011). Repeating fields are less com-

\footnotetext{
Received March 19, 2013; revised 0ct. 20, 2013; accepted 0ct. 24, 2013.

Author contributions:S.L.C. and D.A.N. designed research;S.L.C. and D.A.N. performed research;S.L.C. and D.A.N. analyzed data; S.L.C. and D.A.N. wrote the paper.

This work was supported by grants from the Neurosciences Research Foundation, The G. Harold and Leila Y. Mathers Charitable Foundation, and from DARPA through 0NR Grant N00014-08-1-0728. We thank Drew Maurer, Bryan Wilent, and Jim Kneirim for useful discussion and helpful input regarding the design of figure, and Kara Papaefthimiou for technical assistance with preliminary analyses of tracking and spiking data.

The authors declare no competing financial interests.

Correspondence should be addressed to Dr. Stephen L. Cowen, Department of Psychology, University of Arizona, Life Sciences North Room 347, Tucson, AZ 85724. E-mail: scowen@email.arizona.edu.

DOI:10.1523/JNEUROSCI.1199-13.2014

Copyright $\odot 2014$ the authors $\quad 0270-6474 / 14 / 330232-10 \$ 15.00 / 0$
}

mon near the start and end of a track with repeating elements (Nitz, 2011), and may develop through a gradual learning process (Singer et al., 2010). Together, these results suggest that the mechanism by which the hippocampus encodes space may be more flexible and potentially capable of "chunking" spaces having overlapping sensory or motor context into a common neural code.

The full set of rules yielding the formation and maintenance of repeating fields has not been identified, but commonality in head-direction and path shape appears to be a strong determinant. As such, the comparison of repeating-field responses under varying conditions offers a unique opportunity to identify the contribution of visual cues, inertial/head direction information, maze-shape, and sensory-motor cues that govern spatial firing patterns of hippocampal neurons. Thus, we analyzed the responses of neurons with repeating fields in CA1 as animals traversed a variety of square and circular spiral-shaped mazes in both light and darkness. Across the five loops composing the differently shaped tracks that were used, we demonstrate that repeating fields, under certain conditions, exhibit nonrandom shifts in the angular position of firing fields relative to each other or relative to track center. Backward shifts that likely result from Hebbian plasticity (Mehta et al., 1997) and forward shifts that appear to result from cognitive demands and reward location (Lee et al., 2006) have been reported previously; however, no study has identified or investigated shifts in the center of mass of repeating fields.

The presence of shifts in repeating fields was primarily observed when track-running was associated with continuously changing angular velocity. The forward and backward shifting of 
A





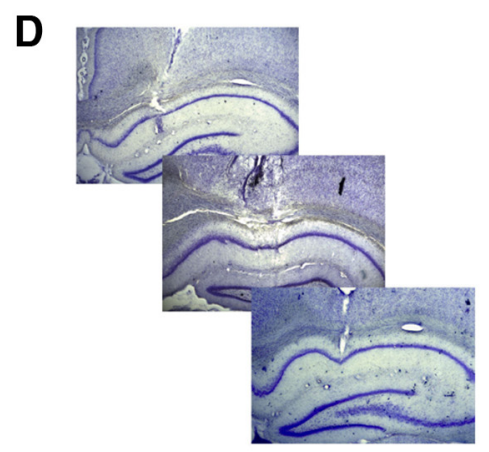

B
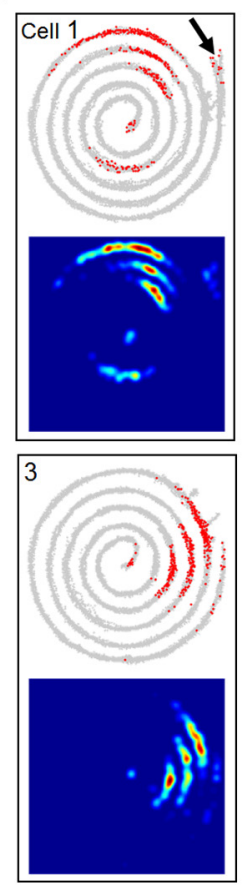

Inward
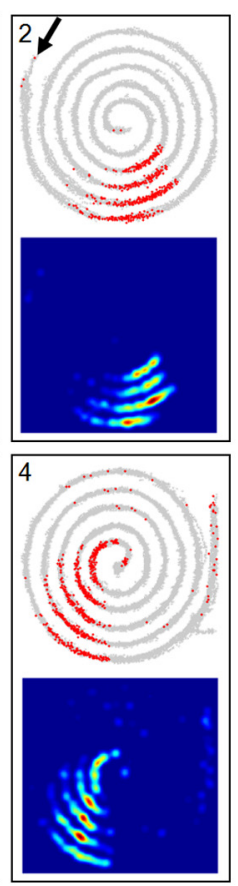

C
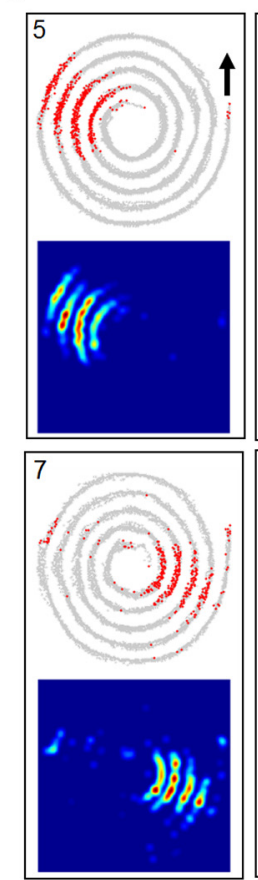

Outward
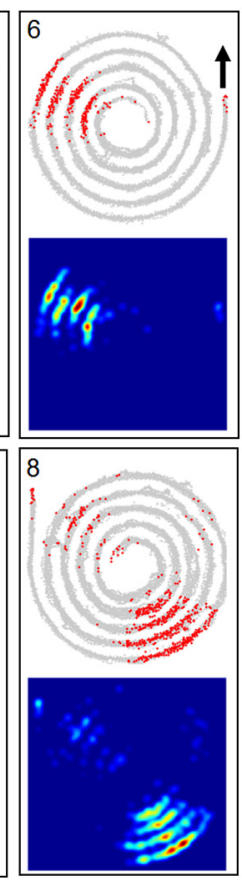

Figure 1. A, Schematic of the circular-spiral track. Each loop of the track is indicated by a color. Animals made multiple inbound and outbound of the track to retrieve a single food reward located at the center or exit point of the track (brown circles). All tracks had $4 \mathrm{~cm}$ high walls that allowed animals to see distal cues along recording room perimeter. $\boldsymbol{B}$, The spiking responses (red dots) and firing rate maps (occupancy normalized density plots) of four neurons that were recorded as animals ran toward the center of the spiral. These examples illustrate the forward shift of the center of mass of each repeating field during the inward journeys. The gray background indicates the position of the animal as measured from the tracking camera. $\boldsymbol{C}$, Same as in $\boldsymbol{B}$, but for outward journeys. D, Representative samples of histological reconstruction of electrode tracks.

repeating fields is associated, respectively, with ever increasing versus ever decreasing angular velocity. Finally, comparison of the degree of field shifting in light- versus dark-running conditions suggests that the extent to which animals use distal cues to correct for errors in self-motion integration also impacts the observed degree of field-shifting.

\section{Materials and Methods}

Subjects/behavior. Recordings were obtained from seven adult, male Sprague Dawley rats. Physiological data from five of these rats was collected for the original Nitz (2011) paper. Animals were trained to traverse three versions of a spiral track (Fig. 1). The tracks were centered on the same position in the recording room. All tracks were composed of five individual "loops" defined by short $(4 \mathrm{~cm})$ walls which permitted visualization of the full recording area and its attendant distal spatial cues. Trajectories differed in shape (squared vs circular spiral vs concentric circles), direction of movement through any given room position, and behavior (left- vs right-turning). Inbound (center-going) and outbound (perimeter-going) path running on each track was motivated by food restriction. Food reward was presented at the center of each spiral for inbound trajectories and at the end of the outermost loop for outbound trajectories. During training, animals also experienced track running under conditions of complete darkness and under conditions where tracks were rotated $180^{\circ}$ relative to their normal position in the room. Before surgery and recordings, experience on all tracks was extensive with daily training occurring over the course of 1-2 months. All procedures fell within the guidelines of the National Institutes of Health and approved Institutional Animal Care and Use Committee protocols.

Measures of movement. Running speed was calculated as the first derivative of the smoothed 2D position data [Savitzky-Golay (polynomial) smoothing: order $=3$, window $=11 \mathrm{~cm}]$. Angular speed was computed from the head angle of the animal measured in relation to the center of the spiral track. This angle was determined by projecting the $2 \mathrm{D}$ position data onto a template of the track and then using the template to deter- mine both the linearized position of the animal and the head angle (Nitz (2011); details regarding the procedure). Measures of the magnitude of linear and angular acceleration were also derived from the measures of speed and angular speed. These values were computed in two steps. First, the derivative of the speed and angular speed signals were identified. Second, the envelope of the absolute value of these values was then determined and used as the measure of acceleration magnitude.

Recordings. Recording electrodes were constructed from polyimideinsulated tungsten wire ( $25 \mu \mathrm{m}$, California Fine Wire). The wire was twisted and fused to form "stereotrode" recording wire pairs, and the electrode tips were gold-plated to impedances of $200 \mathrm{k} \Omega$ and mounted within custom-made microdrives. At the point of connection to a recording tether, signals were current-amplified by an array of field-effect transistors built into the connector assembly (NB Laboratories). Action potentials and local field potentials were amplified (Neuralynx), then digitally recorded using custom-made software courtesy of Matt Wilson (Massachusetts Institute of Technology) and Loren Frank (University of California, Los Angeles).

The animal's position in the room was obtained by tracking head position via an overhead camera which detected LED lights fitted into the recording headstage. Position on the track was then determined by fitting such tracking data to track templates (Nitz (2011); details regarding the procedure).

Single unit discrimination was performed as described extensively in prior publications (Nitz, 2006; Wilent and Nitz, 2007). Subsequent to surgical implantation of microdrive/stereotrode assemblies above the dorsal hippocampus (AP - 3.5, ML 2.2), recordings were obtained within the overlying posterior parietal cortex for $>1$ week. Stereotrodes were then lowered slowly $(\sim 40-80 \mu \mathrm{m} / \mathrm{d})$ until reaching the hippocampus.

Stereotrode locations within field CA1 of the hippocampus were verified by the presence of sharp-wave/ripple events within local field potential recordings. Such in vivo localization was later confirmed by histological analysis of recording wire tracks (Fig. 1). 
Firing field localization. Firing fields were calculated as described by Nitz (2011). To summarize, firing fields were defined as spatially isolated increases in firing rate resulting in peaks exceeding $5 \mathrm{~Hz}$ and persisting at rates of $10 \%$ peak rate or better for at least 15 pixels $(7.5$ $\mathrm{cm})$. The start and end points of firing fields were defined as the positions where firing rate dropped $<10 \%$ the peak firing rate. Firing rates over the range of pixels defined by this method were used to determine the center of mass (COM) of each firing field. To permit comparison of field positions across tracks, it was necessary to also calculate the angle, relative to the recording room, of the COM of firing fields. Note that the use of firing field angles for this analysis presumes that firing field sets bear an allocentric frame of reference as opposed to one based upon the start and endpoints of the track itself.

\section{Results}

Neural recordings ( $n=504$ neurons) were acquired from seven rats as the animals made uninterrupted inbound (i.e., center-going) and outbound traversals on four different tracks (see Materials and Methods). All tracks were composed of five individual loops defined by short (4 $\mathrm{cm}$ ) walls which permitted visualization of the full recording area. Loops were numbered from 1 to 5 (largest-smallest; Fig. 1A). Tracks differed in shape (circular spiral, square spiral, concentric circle), and the total path length of each individual inbound or outbound journey was at least $4 \mathrm{~m}$ for each track.

\section{Forward shift of firing fields during inbound but not outbound traversals of the circular-spiral track}

Observations from a number of laboratories now challenge the notion that hippocampal neurons only respond to single locations within a spatial environment. Results from our own laboratory indicate that $74 \%$ of CA1 principal cells express repeating fields along adjacent loops of the circular-spiral track (Nitz, 2011). In this study, these firing field sets were composed of 3-5 firing-field repeats that appeared along a similar radial angle relative to the center of the track. Visual inspection of these fields suggested that the field centers shifted forward along successive loops of the spiral during inbound traversals (Fig. 1B). The present study analyzed the population of recorded cells for quantitative evidence of such a systematic shift under different conditions. The presence of a systematic shift, despite the presence of clear external landmarks, may provide insight into the integration of self-motion and visual cues as they relate to the spatial firing properties of hippocampal neurons.

To investigate the shift, firing fields acquired from experiments in Nitz (2011) along with fields acquired from two additional rats were analyzed to determine whether the COM of each field-repeat shifted forward or backward during consecutive loops on the track with "forward" and "backward" being defined relative to the animal's direction of motion (see Fig. $2 A$ ). Thus, a forward shift was quantified as a positive angular shift of succes-

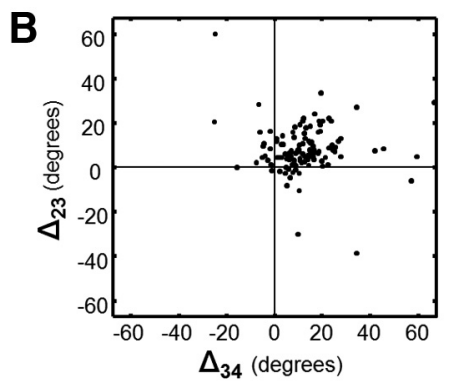

$\Delta_{34}$ (degrees)
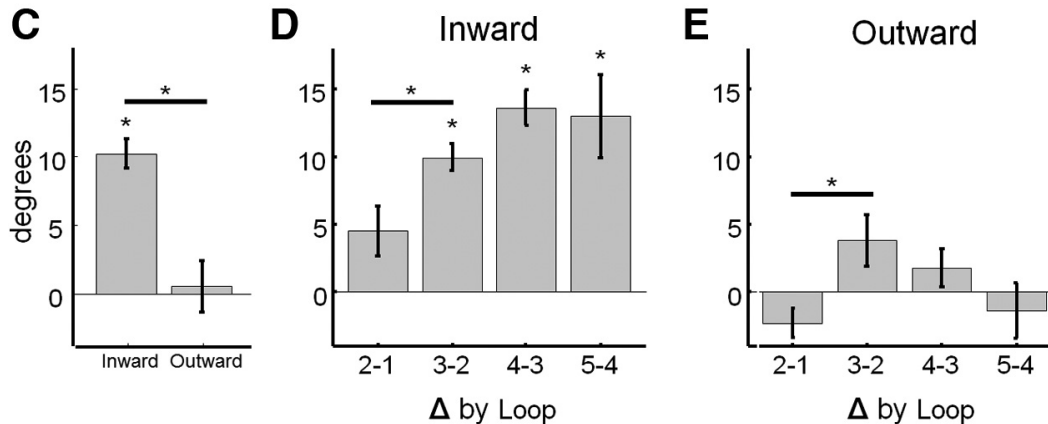

$\Delta$ by Loop

Figure 2. Forward shift occurs during inward but not outward journeys. $\boldsymbol{A}$, Illustration of procedure used for calculating the A forward shift relative to the animal's running direction would be indicated as a positive difference between the centers of mass subfield is identified by the loop in which it was active $B$ Plot of the shift between pairs of adjacent subfields $(n=158)$. Trials were restricted to inward traversals of the spiral. Each firing field is indicated as a point on the plot and the axes indicate the shift loops 2 and 3 ( $y$-axis) and 3 and 4 (x-axis). The majority of field-pairs (114/158) were in the top-right quadrant and 作 zero for outward journeys. Asterisk over the individual bar indicates results of testing for a difference from zero $(t$ test, $p<0.05$, Bo (Tukey-Kramer, $p<0.05$ ). $\boldsymbol{E}$, Same as in $\boldsymbol{D}$ but for outward journeys. No significant shift was observed on a pair of adjacent loops during outward traversals. All error bars indicate SEM.

sive firing-field COMs ahead of the animal regardless of whether the animal was on a journey to the center (inward) or to the outside (outward) of the track. This measure was applied to all neurons exhibiting at least three repeating fields that spanned loops 2-4. The extent of forward and backward shifting under different conditions is visualized by plotting the shift for loops 2 and 3 against the shift in loops 3 and 4 (Fig. 2B). If fields shift forward, points should cluster in the upper right quadrant of the plot. In agreement with what could be observed from the raw positional firing rate plots of Figure 1; a significant portion of the points clustered in the top-right quadrant (114/158 points, $p<$ $0.000001, \chi^{2}$ test, random chance $=158 / 4$ quadrants or 39.5 points).

The magnitude and direction of the shift was analyzed on individual loops of the track (Fig. 2C,D). This analysis indicated a strong forward shift on all adjacent pairs of loops during inward journeys (Fig. $2 C$; $t$ test, $p<0.0125$ on each pair of loops, Bonferroni correction, $n=130$ ). However, no shift was observed for any of the pairs of loops during outward journeys (Fig. $2 D, t$ test, $p>0.0125$, Bonferroni correction, $n=37$ ). To determine why the shift was present on inward but not outward journeys of circular-spiral tracks, we examined the presence/absence of field shifting for a number of track shapes for which repeating fields were observed. 

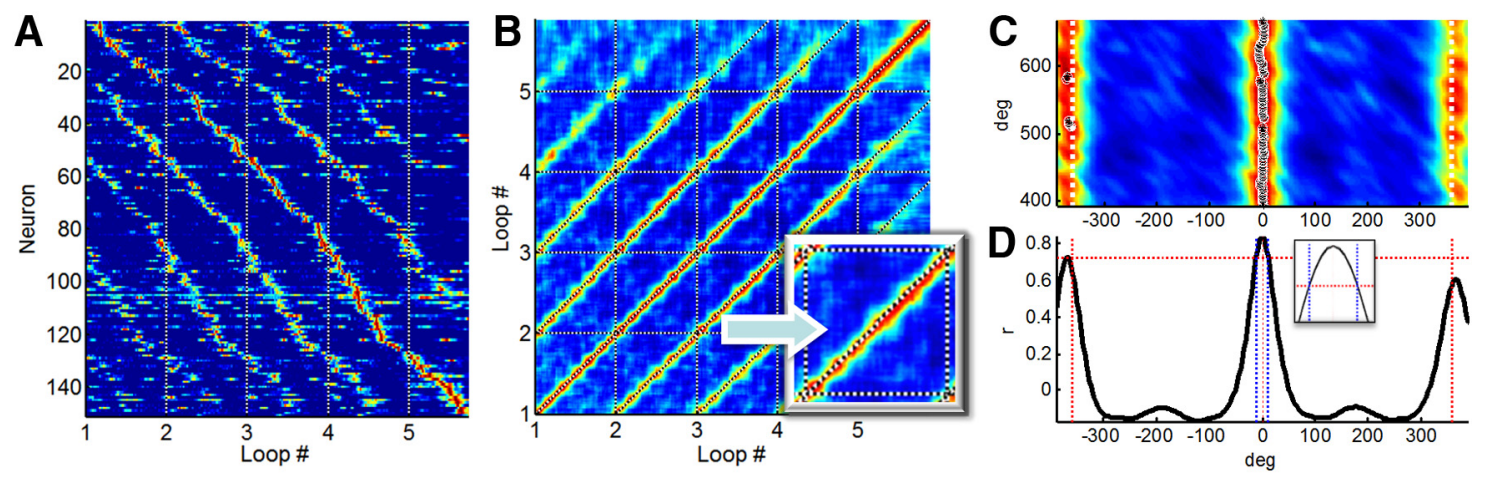

Figure 3. Forward shift observed in population activity. $\boldsymbol{A}$, Firing rate activity of 151 neurons recorded during inward traversals of the spiral track. Each loop (a $360^{\circ}$ rotation) is indicated on the $x$-axis. Inward movement is from the left to the right. $\boldsymbol{B}$, State correlation matrix generated by correlating all population states (the columns in subplot $\boldsymbol{A}$ ) with states at all locations along the track $\left(x\right.$-axis). The matrix is symmetric with respect to the diagonal. Repeating striations along the diagonal indicate that states tended to be correlated with states at $360^{\circ}$ (one loop) intervals. The pattern of striations also suggests that the peak was offset from $360^{\circ}$ by $6.7^{\circ}$ (inset, green box with the diagonal indicated by the dashed line). $\boldsymbol{C}, \boldsymbol{D}$, Reconstruction of position by population state. A reconstruction analysis was performed to determine the fidelity by which populations of repeating fields encoded the current location of the animal relative to other locations ahead of or behind the rat. Template and Test field-by-location matrices similar to subplot $\boldsymbol{A}$ were created from nonoverlapping sets of trials and the similarity of population states (columns) in these matrices were compared (Pearson's $r$ ). Reconstructed position was the state/position with the largest correlation. $\boldsymbol{C}$, Display of state correlations and the reconstructed position (black/white dots) in front of (positive) or behind (negative) the rat for the inner two loops of the spiral. Reconstructed position (black/white dots) clustered at the current location of the rat, and only $5 \%$ of reconstructed positions were outside of $\pm 10^{\circ}$ from the current location. $D$, The column mean and SEM of subplot $C$. Vertical red lines indicate $\pm 360^{\circ}$. The peak correlation at the current location $\left(0^{\circ}\right)$ was $r=0.83$, whereas values at adjacent loops peaked at $\pm 367\left(r_{\text {behind }}=0.71, r_{\text {ahead }}=0.62\right)$. Inset, Close-up of the central peak to illustrate that there was no visible asymmetry in the reconstruction.

\section{Forward shift in the population code}

The presence of a forward shift in the firing responses of individual neurons suggested that shift would also be observable in the activity of populations of neurons (Georgopoulos et al., 1986). Shift in the population response was measured by determining the similarity between population states (the vector of firing rates) at all locations along the track. The presence of repeating fields suggests that such a measure of population-state similarity should repeat at approximately the same angle along subsequent loops. However, the presence of a forward shift in single-unit responses also suggests that the similarity of the current state should be similar to future states that occur slightly ahead of $360^{\circ}$ (one loop traversal). This analysis was performed by first creating a neuron-by-position matrix of firing rates with each column of the matrix being a population state vector (Fig. 3A). Firing activity of each row (neuron) in this matrix was normalized to span a range between 0 and 1 . As with Georgopoulos et al. (1986), the 151 neurons displayed in Figure 3 were recorded across experimental sessions, resulting in an estimate of what a simultaneously recorded population could hypothetically encode. Position in this matrix was measured as the radial position along the spiral with each loop around the spiral being indicated on the $x$-axis $\left(\right.$ bin size $=3^{\circ}$ ). The similarity between population states was measured using the Pearson's correlation coefficient.

Figure $3 B$ presents the state correlation matrix for the 151 neurons recorded during inward traversals of the circular-spiral track. The presence of distinct striations on the off-diagonals of the matrix is in clear accord with the observation of repeating firing fields. Closer inspection of these off-diagonal peaks in the correlation matrix indicated that peaks were offset from the peak angle on the preceding loop (Fig. 3B, inset). This shift in this matrix was quantified by calculating how far the off-diagonal peak differed from $360^{\circ}$. This was determined by rotating the correlation matrix by $45^{\circ}$ and subtracting $360^{\circ}$ from the mean peak value of the first off-diagonal. The off-diagonal peak was shifted forward by $+6.6^{\circ}$. A small negative shift of $-1.8^{\circ}$ was observed for outward journeys which is consistent with the lack of an observed shift in the single-unit analysis.
The same set of neurons used in the preceding analysis was also used to determine the fidelity by which populations of repeating fields encoded the current location of the animal relative to other locations on the spiral such as the corresponding angular position on adjacent loops. Two sets of population states ("template" and "test") were created from nonoverlapping sets of trials and the best match between template and test states was used to determine the reconstructed position of the rat. The population was most effective at encoding the current location of the rat as only $5 \%$ of reconstructed points were outside of \pm 10 degrees from the current position. Even so, there was considerable correlation with locations on adjacent loops at $\sim \pm 367$ degrees, further indicating the presence of a forward shift in the population state.

Angular velocity is largest in the innermost loops of the spiral An important assumption underlying the design of the spiralshaped track was that inward traversals on the track result in the animals experiencing a gradually increasing angular rotational signal. This assumption, however, would be violated if animals exhibit large changes in running speed as a function of loop. To verify that running on the spiral track did indeed result in a gradually increasing angular velocity signal, the rate of angular rotation was calculated for each loop and averaged across sessions and rats (Fig. 4A). This analysis revealed a strong correlation between angular velocity and loop with angular velocity being largest on the innermost loop for both inward and outward journeys $\left(r_{\text {inward }}=0.94 ; p=8.67 \mathrm{e}-168, n=370\right.$ sessions; $r_{\text {outward }}=$ $0.81 ; p=1.3 \mathrm{e}-026, n=110$ sessions). Post hoc ANOVA indicated that all between-loop differences were significant during inward journeys and all differences, with the exception of loops 4 and 5, were significant during outward journeys (ANOVA, $p<0.05$, Tukey-Kramer correction). A weaker but significant correlation was also found between loop and running speed (Fig. 4B) with speed being lowest on the innermost loops $\left(r_{\text {inward }}=-0.43 ; p<\right.$ $\left.1 \mathrm{e}-17, n=370 ; r_{\text {outward }}=-0.71 ; p<6 \mathrm{e}-18, n=110\right)$. In contrast to angular velocity, post hoc analysis of running speed only identified differences between speeds on loops 3-4 and 4-5 on inward journeys and between 1-2 and 4-5 on outward journeys (ANOVA, $p<0.05$, Tukey-Kramer correction). 


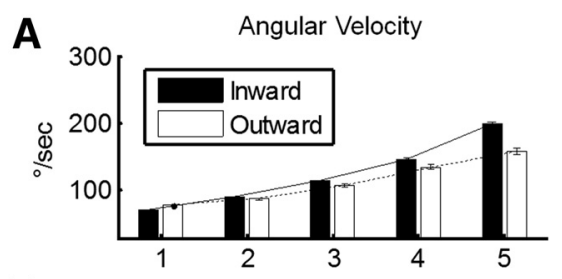

B

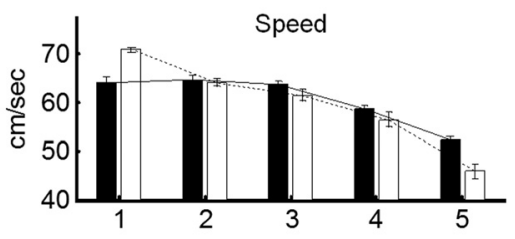

C

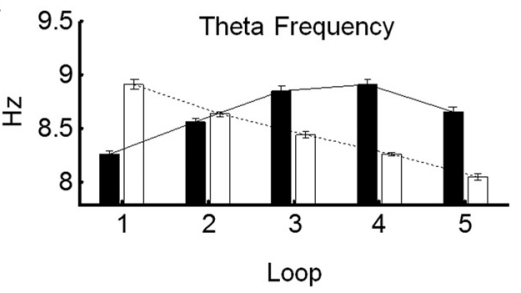

Figure 4. Angular velocity and running speed change as a function of loop. On each experimental session, the average angular velocity and speed were determined for inward and outward journeys. $\boldsymbol{A}$, Angular velocity (degrees/s) was a function of distance to the center of the spiral, being smallest on the outermost loops and largest on the innermost loops (loop 5). There was a significant positive correlation between angular velocity and loop on both inward (loops $1-5$ ) and outward (loops 5-1) journeys $\left(r_{\text {inward }}=0.94 ; p=8.6672 \mathrm{e}-168, n=370\right.$ sessions; $r_{\text {outward }}=0.81 ; p=1.2998 \mathrm{e}-026, n=110$ sessions). $\boldsymbol{B}$, A weaker but significant correlation between running speed and loop number was also observed for inward (ANOVA, $p<1 \mathrm{e}-17$, $n=75$ sessions) and outward journeys (ANOVA; $p<5 \mathrm{e}-16, n=22$ sessions). There was also a significant negative correlation between running speed and loop number $\left(r_{\text {inward }}=-0.43\right.$; $\left.p<1 \mathrm{e}-17, n=370 ; r_{\text {outward }}=-0.71 ; p<6 \mathrm{e}-18, n=110\right)$. Post hoc analysis of running speed indicated that there was no significant difference between loops 2 and 3 . Together, the running on the spiral track most strongly affected angular velocity, although significant changes in running speed were also observed. C, The frequency of the hippocampal theta rhythm often increases with increasing running speed. No clear connection between theta frequency and running speed or angular velocity was observed during traversals of the spiralshaped track. In fact, theta frequency tended to increase as rats ran inward and increase as rats ran outward $\left(r_{\text {inward }}=0.36 ; p<1 \mathrm{e}-12, n=366 ; r_{\text {outward }}=-0.88 ; p<1 \mathrm{e}-40, n=135\right)$ despite opposite patterns of speed and angular velocity $(\boldsymbol{A}, \boldsymbol{B})$. All error bars indicate SEM.

The frequency of the theta rhythm in the hippocampus has been found to increase with running speed, typically increasing in frequency with increases in speed (McFarland et al., 1975; Rivas et al., 1996; Sławińska and Kasicki, 1998). To determine whether this pattern was observed on the spiral track, we also calculated the average theta frequency as a function of loop. Contrary to such a prediction, no clear association between theta frequency and running speed or angular velocity was observed. In fact, theta frequency increased as rats ran inward (decreasing running speed, increasing angular velocity) and increased as rats ran outward (increasing running, decreasing angular velocity; Fig. $4 A, B)$. This pattern was identified through a regression of theta frequency and speed $\left(r_{\text {inward }}=0.36 ; p<1 \mathrm{e}-12, n=366 ; r_{\text {outward }}=\right.$ $-0.88 ; p<1 \mathrm{e}-40, n=135)$.

\section{Summary of hypotheses}

Forward shifts in the centers of mass of repeating place fields have not been previously reported using tracks that did not have spiral shapes (Derdikman et al., 2009; Singer et al., 2010; Hayman et al., 2011). The lack of previous reports combined with the observation that running on the spiral results in gradually increasing (inward journeys) or decreasing (outward journeys) angular ve-

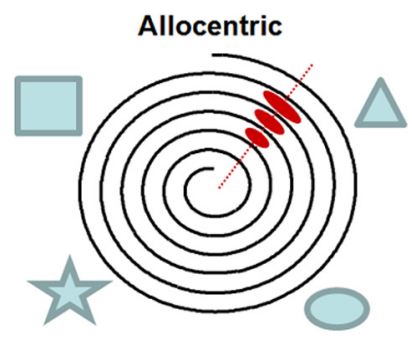

A
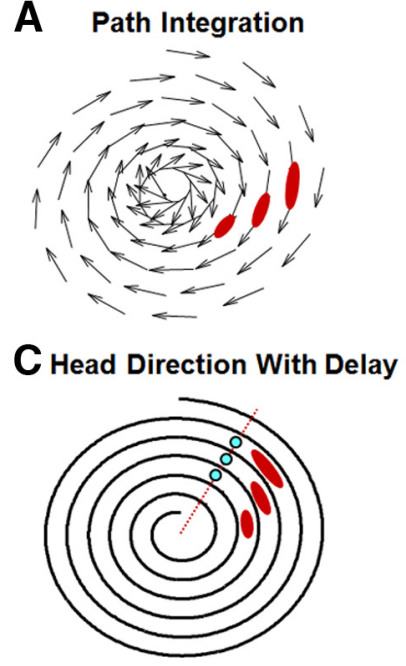

B Local View



D Step Counting

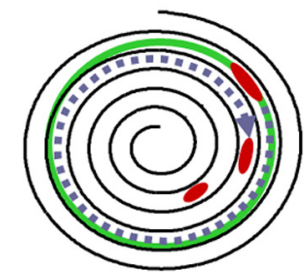

Figure 5. Summary of hypotheses: the observed forward shift suggests that firing activity was not anchored in a purely allocentric reference frame (top plot) as such anchoring would predict that the fields would fire at the same angle on consecutive loops. Four potential mechanisms for the shift were explored. $\boldsymbol{A}$, Path integration error: rats can navigate in darkness by integrating self-motion cues such as head rotation and running speed. The spiral is unique in that angular rotation gradually increases during the inward journey. The slow rate of change may cause animals to underestimate the amount of angular distance traveled and produce a forward shift. $\boldsymbol{B}$, Local view: a shift in the firing field on the spiral could occur if neurons fired when animals spot a specific visual cue or local view. In this illustration, the cell fires at the time at which animals viewed a cue mounted on a wall outside of the maze. Contrary to our observations, however, this hypothesis predicts a backward shift, as animals would view the cue at earlier points on consecutive laps (presuming the animals look forward at an angle tangent tot the track). C, Head direction at fixed delay: a forward shift could result from neurons responding in a delayed manner to a head direction experienced in the recent past (e.g., if the neuron is receiving a delayed head-direction signal from an afferent neuron). A fixed temporal delay between the responses would allow the animal to cover more angular distance on the inner relative to the outer loops before the cell would fire. $\boldsymbol{D}$, Step counting: in addition to path integration by integrating angular rotation and running speed $(\boldsymbol{A})$, animals could also path integrate by "counting steps" along the spiral. Step counting would produce a forward shift if the animal worked under the assumption that the spiral was a concentric circle (green circle).

locity signals (Fig. $4 A$ ) suggest that the shape of the track contributed to the shift. The following section summarizes ways in which the track and this rotational signal may produce the shift. These hypotheses are explored through experiments using different tracks and light/dark conditions. Subsequent sections will also address the interesting observation that forward or backward shifts in place-field centers were not observed during outward journeys (Fig. 2C).

We explored four potential sources of the shift (summarized in Fig. 5). First, the shift could result from path-integration errors amplified by the rotational input produced by the spiral track (Fig. 5A). Rotational signals are powerful drivers of hippocampal activity as suggested by the observation that head-direction cells compose one of the of the principal inputs to CA1 (Sargolini et al., 2006). This input is a vital component of the navigation sys- 
tem when visual cues become unreliable. In the absence of external cues, reliance upon self-motion input, such as head-direction signals, results in the gradual buildup of error (McNaughton et al., 1996). Such error may be expressed in the shifting of field centers. Second, the shift could result from differences in the animal's local view of landmarks at different locations along the track (Fig. 5B). For example, a shift in the place field could occur if neurons fired at the time at which animals spotted a specific visual cue on the wall. This hypothesis can be immediately rejected, however, as responses to a local view would predict a backward shift as animals would view the cue at earlier points on consecutive laps, presuming the animals look forward at an angle tangent to the track (Fig. $5 B$, green lines). Third, the shift could result from neurons responding in a delayed manner to a specific head direction (Fig. 5C). For example, the CA1 neuron could potentially receive a delayed signal from the head direction system. A fixed temporal delay such as this would result in the animal to covering more angular distance on the inner loops before activation of the CA1 neuron, producing a forward shift on the inner loop. Finally, animals could estimate their location using a step counting strategy. In one version of this strategy, animals could gauge their radial distance to the center of the track from their current location and then estimate the radius and circumference of a circle at this point. Step counting could be used to judge progress round the circumference of this estimated circle. The gradual reduction of the radius of the spiral-shaped path during inward journeys would result in animals underestimating the amount of radial distance traveled, producing a forward shift (Fig. 5D). The following sections explore these hypotheses.

\section{Forward shift is eliminated under conditions of constant or zero angular acceleration}

In the study by Nitz (2011), repeating firing fields were observed under conditions of darkness, suggesting that fields can be driven by self-motion input. Given the strong self-motion input into CA1 and the presence of head-direction cells in the medial entorhinal cortex (Sargolini et al., 2006), it is possible that the shift resulted from the increasing angular velocity signal produced by running on the track. To determine whether angular input was a source of the forward shift, animals were trained to run on two tracks that restricted angular acceleration while maintaining the five-loop structure of the original circular-spiral track.

The first track consisted of a square-shaped spiral. Journeys on the linear sections of this track have linear but no angular acceleration (Fig. 6A,E). Consequently, no shift should be observed on this track if angular input is the principal contributor to the forward shift. Indeed, no shift was observed during inward journeys on the square-shaped spiral track ( $p=0.33, n=416$ fieldpairs from 140 neurons). A second track was also constructed which consisted of a series of progressively smaller concentric circles (Fig. 6B,E). Animals entered adjacent inward loops of the track through short junctions (see Fig. 6B). Critically, traversals within a loop on this track will have a constant as opposed to continuously increasing angular velocity signal. Repeating fields were analyzed during inward traversals, and repeating fields with centers within $20^{\circ}$ from the junctions were omitted from the analysis given the possibility for contamination from movements or cues related to traversing the junctions. Results from the analysis of field centers on the concentric track indicated a very small mean positive shift of $4^{\circ}$ for all successive loop pairings (Fig. $6 E$; $p=0.041, n=179$ field-pairs from 51 neurons) which was significantly lower than the $10^{\circ}$ shift observed on the spiral $(t$ test, $p<0.05)$. To summarize, the elimination or significant reduc-

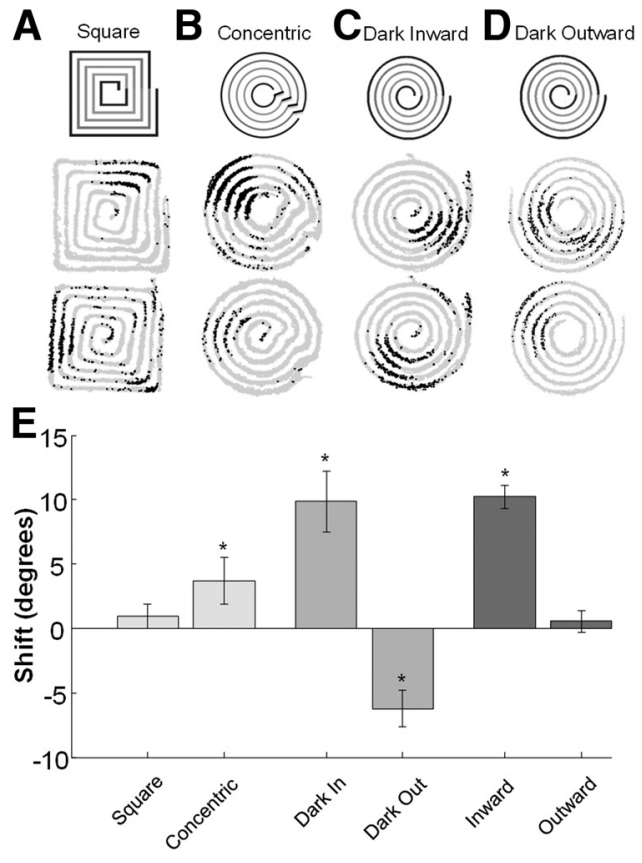

Figure 6. Degree of shift during traversals on concentric-circle and square tracks and in darkness. $\boldsymbol{A}$, Schematic illustration of the squared spiral track and the spiking responses of two neurons during inward traversals on this track. $\boldsymbol{B}$, Same as in $\boldsymbol{A}$ except for the concentric circle spiral track. The field in the upper plot did not show a clear forward shift, whereas a forward shift was observed in the field on the lower plot. C, D, Examples of responses from neurons during inward and outward journeys on the circular spiral in the dark. $\boldsymbol{E}$, The degree of shift during successive loops of various tracks. The $y$-axis indicates that average shift in the center of mass of fields on adjacent loops (Fig. 2A). Data were pooled across all loops $\left(\Delta_{12}, \Delta_{23}, \Delta_{34}\right.$ and $\left.\Delta_{45}\right)$. Positive values indicate that the fields shifted ahead of the animal on each consecutive loop. All tests for deviation from zero were performed using two-tailed $t$ tests. All reported $P$ values are unadjusted. Bar labels: Square, No significant shift ( $p=0.33, n=416$ field-pairs from 140 neurons) was observed on the square-spiral track; Concentric, A small but significant forward shift was observed ( $p=0.041, n=179$ field-pairs from 51 neurons) during inward journeys on the concentric circle track; this shift was significantly lower than the $10^{\circ}$ shift observed on the spiral ( $t$ test, $p<0.05)$; Dark in, Significant forward shift ( $p=0.0001, n=38$ comparisons from 11 neurons); Dark out, Fields shifted backward ( $p=0.00003, n=72$ fieldpairs from 39 neurons); Inward, Outward, For comparison, the magnitude of the shift present during inward and outward journeys on the circular-spiral track are also presented (identical to plot in Fig. 2C).

tion of a forward shift during traversals of two spiral-shaped tracks which minimized continuous changes in angular velocity (i.e., continuous angular acceleration) suggests that the forward shift is largely the result of a persistently increasing angular acceleration signal.

On spiral tracks, angular acceleration is both a function of the location of the rat on the spiral (Fig. 4) and the rat's running speed. For example, at a given location on the spiral track, faster running speeds yield greater linear and angular acceleration at that location. There was a small degree of intertrial variation in running speed on the track $(\mathrm{SD}=2.77$ $\mathrm{cm} / \mathrm{s}$ ). As a result, it was conceivable that an analysis of the COM of firing fields generated during faster-running trials could exhibit a forward shift relative to fields generated during relatively slower trials. To test this prediction, a median split of trials was performed based on running speed. The COM of individual fields within a repeating field was created and compared for the fast and slow groups. Analysis was restricted to single fields located on loops 2, 3, or 4 . No difference in the COM between fast and slow trials was observed (Fig. $7 ; p=$ 


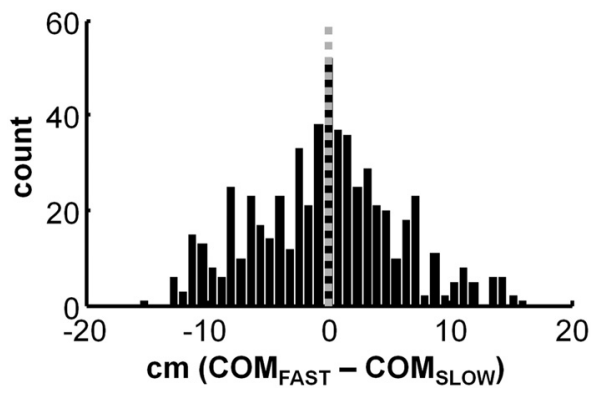

Figure 7. Analysis of the relationship between running speed and the forward shift. Trials were divided in to fast and slow trials according to running speed and firing fields were generated for these two groups. The centers of mass of these fields were compared. The histogram plots the distribution of differences of the field centers in between fast and slow trials $\left(\mathrm{COM}_{\text {FAST }}-\mathrm{COM}_{\text {SLOW }}\right)$. No significant effect of running speed was observed ( $p=0.32, t$ test, $n=628$ fields). Analysis was restricted to fields on loops $2-4$.

$0.32, n=628$ individual fields). This result could indicate that factors beyond acceleration contribute to the shift; however, the lack of an effect could also have been due to a lack of statistical power. First, rats were well trained and, as a result, intertrial variations in running speed were small $(\mathrm{SD}=2.77$ $\mathrm{cm} / \mathrm{s}$ where mean speed was $\sim 60 \mathrm{~cm} / \mathrm{s}$ ). As a result, the range of available speeds may not have been sufficient to identify an effect at the resolution of trials. Second, the median split reduced the number of trials used to compute firing fields (and the COM) in half, resulting in only four to seven trials for each COM estimate, reducing the capacity to accurately estimate the shift.

\section{Forward shift is present when rats completed inward and} outward traversals of the circular-spiral track in darkness

The constellation of visual cues and landmarks within an environment is a powerful anchor for spatial firing activity within the hippocampus (O'Keefe, 1976). Even so, place fields can be maintained in complete darkness through the integration of self-motion information (McNaughton et al., 1983a; Quirk et al., 1990) and self-motion cues may sometimes outweigh distal visual cues as determinants of firing position (Gothard et al., 1996). It is therefore conceivable that forward shifts are due to the buildup of path-integration error. Yet, such an account would fail to explain the lack of field shifts for outward journeys on the circular-spiral track unless animals switched from the use of ideothetic to distal landmark information during inward versus outward journeys. We therefore predicted that fields should shift backward during outward journeys on the circular-spiral in darkness as darkness would force animal to use path-integration strategies for navigation.

To test this prediction, rats were trained to run inward and outward journeys of the circular-spiral track in darkness. In correspondence with the prediction, firing fields shifted forward during inward journeys (as they do in the light) and backward during outward journeys (Fig. $6 E$; inward: $t$ test, $p=0.0001, n=$ 38 comparisons from 11 neurons; outward: $p=0.00003, n=72$ field-pairs from 39 neurons). These results further support the hypothesis that the field-shift is due to intrinsic signals from path-integration systems. This result also suggests that hippocampal input on the inward journeys of the spiral is driven by self-motion and not allocentric cues.

Forward shift is not a direct result of a step-counting strategy The previous analysis demonstrated that the field shift was not due to visual cues and suggests that self-motion signals contrib-

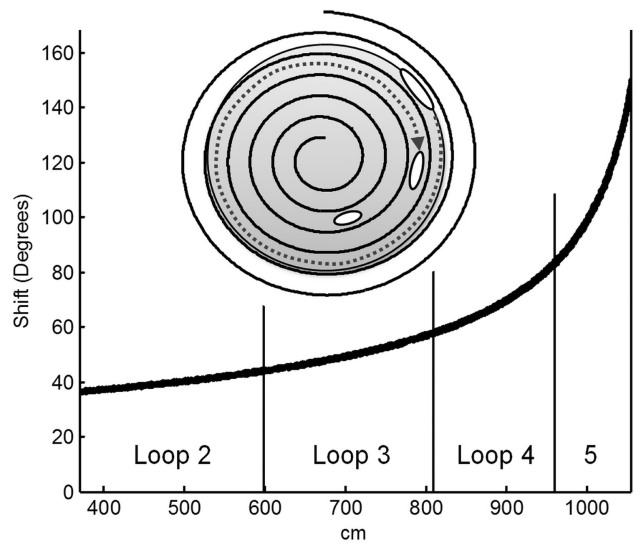

Figure 8. Step counting. One way that shift could develop is if self-motion signals from the motor system were used to integrate distance traveled along the spiral track. If animals were capable of estimating the circumference of the track at each location (shaded circular area in inset), then this strategy could provide an estimate regarding when animals would complete a $360^{\circ}$ rotation. Running on the spiral-shaped track would induce an error in this estimate as the radius of the spiral gradually decreases, thus the estimated circumference would overestimate the distance required to return to the original heading (dashed line). The magnitude of this error was estimated by first determining the circumference of a circle at each point on the spiral and then projecting this distance onto the trajectory taken by animals along the spiral. This projection resulted in a large angular shift that increased exponentially during inward traversals. Most notable was the fact that this estimate of shift was far greater than the $10-15^{\circ}$ shift observed on the spiral. Furthermore, the observed shift did not increase exponentially in the manner indicated by the step-counting estimate. This suggests that the forward shift was not a firstorder function of integrating steps.

ute to the forward shift. There are, however, multiple forms of self-motion input into the hippocampus. For example, in addition to input from the head direction system, the hippocampus also receives self-motion input from motor and somatosensory systems. Such signals could be used like a pedometer to estimate distance traveled along the spiral based on integrating steplengths. Such a measure would estimate an equal travel distance per step. Animals could estimate the distance in steps required to complete a lap around the spiral based on estimating the distance required to travel around the circumference of a circle that has a radius estimated from the current radial distance to the center of the track (Fig. 8, inset). Because the spiral gradually reduces in radius, such a distance estimate would result in a forward shift, as estimates based on the projected circumference would overestimate position on the spiral.

To gauge whether behavior corresponded to such a strategy, we estimated the degree of expected forward shift by first measuring the circumference of concentric circles centered on the track and with radii equal to each position along the spiral (Fig. 8, inset). The distance around these circles was projected onto the spiral track to estimate the degree of shift. Two observations suggest that step counting is not the main contributor to the forward shift. First, we observed that the magnitude of the estimated shift ranged between 40 and $160^{\circ}$ (Fig. 8). These values were far larger than the $10-15^{\circ}$ shift observed on the spiral (Fig. $2 D$ ). Second, the step-counting estimate predicted an exponential increase in shift during the journey to the center of the spiral. No exponential increase was observed in the behavioral data (Fig. 2D). It is conceivable that step-counting information is transformed within or outside of the hippocampus to account for these two observed discrepancies; however, these discrepancies do argue against a straightforward application of step counting to the observed forward shift. 
A

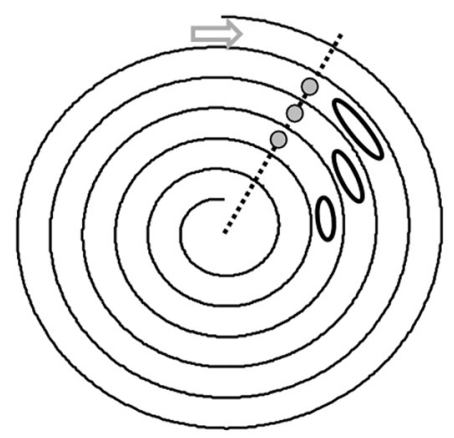

B

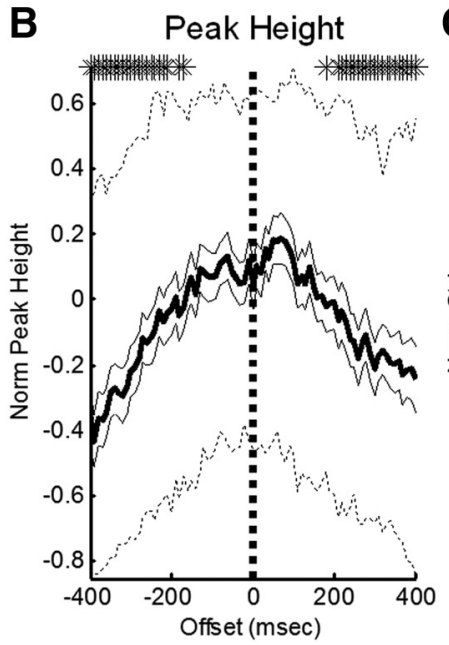

C

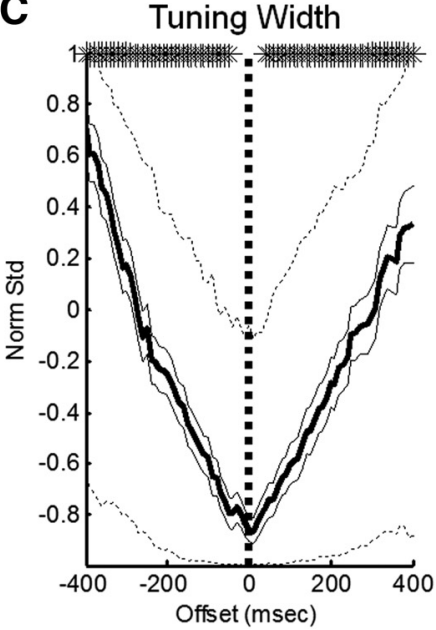

D

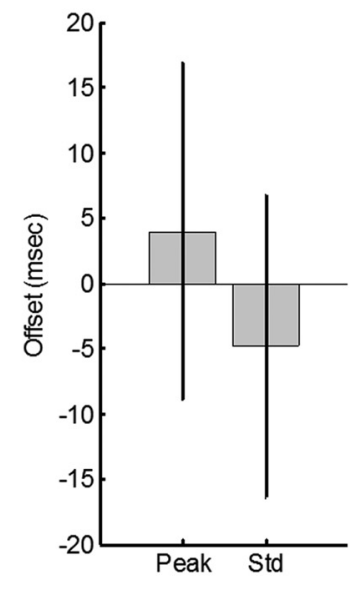

Figure 9. A, Illustration of how a forward field shift during inward journeys could result from a delayed response of neurons to a head angle experienced in the recent past. The dashed line indicates the angle at which a neuron in a structure upstream of the hippocampus may be tuned. If there is a delay in the response of hippocampal neurons to such a cell (e.g., due to transmission or processing delays), then the angular response measured in the hippocampus would appear to shift forward as animals would cover more angular distance per unit time on the inner as opposed to the outer loops. If neurons with forward shifting fields are responding to previously experienced head angles, then measures of tuning quality such as the peak and width of the tuning response to head angle should become optimal for a point in time in the past. To examine this hypothesis, the peak and width of the firing fields were examined after time shifting the spike trains by varying temporal offsets (10 ms increments). The best tuning response should be indicated as the offset with the highest peak and most narrow width. The analysis was restricted to individual firing fields recorded during inward journeys on laps $2-4$ of the spiral ( $n=391$ fields, 110 neurons). $\boldsymbol{B}$, The peak of the tuning curve calculated at different temporal offsets. Thick lines indicate median values of the peak heights at each offset (rescaled to the range of -1 to $1 ; y$-axis). Thin lines indicate the notch confidence interval [ $\pm 1.58 \mathrm{IQR} / \mathrm{sqrt}(n)$ ], whereas the dashed lines indicate the 15.9 and 84.1 percentiles (spanning $68.2 \%$ of the data). Asterisks indicate median values that differed significantly from the median at an offset of zero ( $p<0.05$, rank sum test). No significant shift from a zero offset was observed in the peak. C, The same analysis was performed on the width of the tuning curves (width was quantified as the SD). As with the peak, no clear offset in the minimum of the tuning width was observed. As with peaks, widths were rescaled to range from -1 to 1 . D, The offset associated with the maximal peak height and minimum width (SD) was computed for each field. These means of these values were not different from zero ( $t$ test, $P_{\text {peak }}>0.75, P_{\mathrm{SD}}>0.67, n=391$, error bars $=\mathrm{SEM}$ ).

\section{Forward shifting firing fields are tuned to the animal's current location}

The forward shift could result from firing fields responding with a delay to previously visited locations (Fig. 5C). For example, if a neuron responded to a radial angle visited $100 \mathrm{~ms}$ in the past, the field would appear to shift forward during successive inward loops as animals would cover more angular distance during the $100 \mathrm{~ms}$ interval on the inner loops compared with the outer loops. Delays on the order of $100 \mathrm{~ms}$ could produce the $10 \mathrm{~cm}$ forward shift in field centers given the observed angular speeds on loops 2, 3, and 4 of 90, 115, and 150 degrees/s, respectively. Given these speeds, a temporal delay of $100 \mathrm{~ms}$ would shift fields on these loops forward by 9.0, 11.5, and 15.0 degrees $($ mean $=11.8$ degrees). Delays on the order of $100 \mathrm{~ms}$ are quite long with respect to delays introduced by axonal conduction. For instance, a recent study by Zhang et al. (2013) used antidromic optogenetic stimulation of CA1 neurons to trigger spikes in the medial entorhinal cortex, the most proximal source of head-direction input to CA1. Latencies reported in this study were $<10 \mathrm{~ms}$, a factor of 10 smaller than the $100 \mathrm{~ms}$ estimate. As a result, delays on the order of $100 \mathrm{~ms}$, if present, would likely be due to multisynaptic processes either within or between regions.

The hypothesis that temporal delays in the arrival of headdirection information to CA1 drives the forward shift was explored by determining whether neural tuning response to locations on the spiral had the greatest selectivity for the current location or for locations visited in the recent past or future. Selectivity was measured as the peak height and the tuning-curve width with larger peaks and narrower widths being indicators of improved tuning. These measures were computed by recalculating the tuning curves of individual neurons at different temporal offsets (by adding or subtracting from the original spike times) ranging from -400 to $+400 \mathrm{~ms}$ (increment, $10 \mathrm{~ms}$ ). Analysis of the peak and width of the firing fields at different offsets did not reveal any significant bias in peak or width for times in the past or future (Fig. 9). Specifically, no peak in the tuning curve was observed to significantly exceed the value observed at an offset of zero and no tuning width was observed to fall below the value at an offset of zero (Fig. 9C; Wilcoxson rank sum test, $p<0.05$ ). Together, these results suggest that repeating fields responded with greatest fidelity to the rat's current location and that the forward shift in the center of mass is not due to temporal delays in the arrival of head-angle information into the hippocampus.

\section{Discussion}

The presence of repeating firing fields across multiple loops of spiral-shaped and concentric-circle tracks presented a clear opportunity to examine the effects of idiothetic, or "self-motion" input in driving the location-specific firing of hippocampal neurons. In this study, we identified conditions by which successive repeating fields shifted forward on each successive loop of a spiral-shaped maze. These shifts differ significantly from shifts in the center of mass of hippocampal neurons reported in other studies. For example, place field expansion (Mehta et al., 1997) is a small $(\sim 1.4 \mathrm{~cm})$ backward shift in the center of mass of fields that occurs during the first few trials of a task and may result from Hebbian plasticity. Large forward shifts have been reported more recently in an alternation task where shifts moved progressively toward reward locations (Lee et al., 2006). These shifts, however, occurred gradually across all trials, were present on linear tracks, and were far larger $(\sim 30 \mathrm{~cm})$ than the shifts observed in the present study.

The pattern by which the center of mass of repeating fields shifted on inbound and outbound traversals of circular-spiral, squared-spiral, and concentric-circle tracks suggested that continuous change in angular velocity (angular acceleration) was a 
leading contributor to the observed forward shift. The small but significant shift observed on the concentric spiral also suggests that constant angular speeds also contribute (but to a lesser degree) to the forward shift. Continuous increases in angular velocity, which were encountered only on inbound traversals of circular-spiral tracks, were associated with forward shifts in the field center relative to the analogous firing fields on preceding loops. Consistent with a role of angular velocity in driving this effect, such shifts: (1) were not observed for squared-spiral tracks which are associated with an angular velocity of zero, (2) were exceedingly small for concentric tracks where angular velocity within a loop is constant, (3) could not be easily explained by a "step counting" form of path integration, (4) were not accounted for by a time-delayed responses of hippocampal neurons to headdirection information, (5) would be opposite in sign according to a distal-view model for hippocampal place-specific firing, and (6) most importantly were, as expected, opposite in sign (i.e., backward-shifting) when visual landmarks were unavailable during outbound traversals of circular-spiral tracks which was associated with steady decreases in angular velocity. Despite the weight of evidence favoring the interpretation that changes in angular velocity are particularly effective in producing shifts in the precise placements of repeating fields, it must be acknowledged that the present analyses do not rule out a contribution of step-counting and/or temporal delay in head direction information to field placements. Given the structure of the circular spiral tracks, error in self-motion integration using step counting would be expected to yield exponentially increasing shifts as animals traversed the five loops. This was not observed. However, it is possible that positional updating based on step counting follows a nonlinear function such that this form of self-motion integration explains, in part, our present results. Future studies are required to determine the extent of these additional factors.

Recent work demonstrates that accumulated errors in the tuning of head-direction neurons of the anterodorsal thalamus are directly related to errors in navigation in the dark (Valerio and Taube, 2012). Given this and the fact that head-direction cells are sensitive to linear and angular velocities and can be predictive of upcoming head orientations (Blair and Sharp, 1995; Stackman and Taube, 1998; Taube and Muller, 1998; Cho and Sharp, 2001), it seems possible that the firing field shifts of our CA1 neurons are secondary to alterations in the firing of head-direction cells. If so, then the present results would predict field shifts for grid cells of the medial entorhinal cortex whose patterned spatial firing appears to depend in part on head direction cells, and which itself represents that most likely source for integration of self-motion information (McNaughton et al., 2006; Sargolini et al., 2006). The present results also suggest that the rate of degradation in spatial specificity of grid cell firing in the dark (Hafting et al., 2005) should correlate with the degree of variability in angular velocity.

It is interesting to consider that the presence of stable local landmarks on the square-spiral track (the corners) and the circular-spiral track (the short pathways to the next circle) served as "reset" points by which the hippocampal/entorhinal map could be realigned to the allocentric reference frame. Such realignments could account for the absence of a shift on the square spiral and the near absence on the concentric mazes. This possibility is suggested by a recent study of hippocampal and entorhinal activity in rats traversing a zigzag shaped maze with multiple repeating sections (Derdikman et al., 2009). Under these conditions, the hippocampal and entorhinal networks appear to become reset when animals perform sharp turns. That is, the network was realigned relative to the position of the turn itself. A unique feature of the circular-spiral track is the absence of such behavioral inflection points, and without such local landmarks, path-integration input may drive the spatial organization of the repeating fields. It will be of interest in future work to determine whether the introduction of behavioral inflection points, such as full stops to obtain reward, would, if aligned to analogous positions on each loop, yield an absence of field shifts.

The lack of evidence for significant shifts in field centers during outbound traversals of circular-spiral tracks in the light appears, at first glance, to be at odds with the claim that continuous changes in angular velocity are important contributors to the observed shift. However, robust backward shifting of firing fields was observed during outbound traversals of circular-spiral tracks in the dark. Because the primary difference in running in light versus dark concerns availability of distal visual landmarks and since, such landmarks controlled hippocampal spatially specific discharge in these same animals (Nitz, 2011), a likely explanation of the full set of findings concerning field shifts on circular-spiral tracks is that updating of the hippocampal mapping of position is primarily dependent on integration of self-motion information for both inbound and outbound runs in the dark as well as inbound runs in the light. In contrast, updating of the hippocampal mapping of position during outbound runs in the light appears to be equally dependent on the use of the animal's spatial relationship to the constellation of distal visual landmarks. Following this explanation further, we suggest that the differences in the positioning of the reward site on inbound versus outbound traversals may be key to the observed differences in updating of the hippocampal map of environmental space. As each spiral track was centered within the array of distal landmarks, inbound runs required animals to circle the goal location during inward journeys. As a result, the goal location lacked a consistent reference frame in allocentric space. In contrast, the goal site for outbound runs lied within a single quadrant of the environmental space, which was constant regardless of where the animal was located on the spiral. Because of this, it may be possible for the animal to more easily use its position relative to visual landmarks to gauge its position relative to the goal site on outbound versus inbound runs. As a consequence, outbound runs in the light may have been subject to frequent correction of hippocampal updating of position based on path integration by reference to position relative to distal landmarks. Although admittedly speculative, the above reasoning can fully explain the distinct differences in field shifting observed for the different types of spiral track traversal used in the present work. Furthermore, prior work has demonstrated that firing patterns of hippocampal neurons can be simultaneously influenced by the animal's position relative to a goal and the animal's position with the larger environmental space (Gothard et al., 1996).

Additional work is required to pin down the cellular and/or network mechanisms responsible for the shift observed in repeating fields. Nevertheless, the present results indicate that changing angular velocity can directly impact the updating of hippocampal spatial representations and demonstrate that effective design of track-running environments can help to unlock the neural mechanisms by which very different sources of spatial information interact to generate the brain's representation of environmental space.

\section{References}

Blair HT, Sharp PE (1995) Anticipatory head direction signals in anterior thalamus: evidence for a thalamocortical circuit that integrates angular 
head motion to compute head direction. J Neurosci 15:6260-6270. Medline

Cho J, Sharp PE (2001) Head direction, place, and movement correlates for cells in the rat retrosplenial cortex. Behav Neurosci 115:3-25. CrossRef Medline

Derdikman D, Whitlock JR, Tsao A, Fyhn M, Hafting T, Moser MB, Moser EI (2009) Fragmentation of grid cell maps in a multicompartment environment. Nat Neurosci 12:1325-1332. CrossRef Medline

Georgopoulos AP, Schwartz AB, Kettner RE (1986) Neuronal population coding of movement direction. Science 233:1416-1419. CrossRef Medline

Gothard KM, Skaggs WE, Moore KM, McNaughton BL (1996) Binding of hippocampal CA1 neural activity to multiple reference frames in a landmark-based navigation task. J Neurosci 16:823-835. Medline

Hafting T, Fyhn M, Molden S, Moser MB, Moser EI (2005) Microstructure of a spatial map in the entorhinal cortex. Nature 436:801-806. CrossRef Medline

Hayman R, Verriotis MA, Jovalekic A, Fenton AA, Jeffery KJ (2011) Anisotropic encoding of three-dimensional space by place cells and grid cells. Nat Neurosci 14:1182-1188. CrossRef Medline

Lee I, Griffin AL, Zilli EA, Eichenbaum H, Hasselmo ME (2006) Gradual translocation of spatial correlates of neuronal firing in the hippocampus toward prospective reward locations. Neuron 51:639-650. CrossRef Medline

McFarland WL, Teitelbaum H, Hedges EK (1975) Relationship between hippocampal theta activity and running speed in the rat. J Comp Physiol Psychol 88:324-328. CrossRef Medline

McNaughton BL, Barnes CA, O'Keefe J (1983a) The contributions of position, direction, and velocity to single unit activity in the hippocampus of freely-moving rats. Exp Brain Res 52:41-49. Medline

McNaughton BL, O'Keefe J, Barnes CA (1983b) The stereotrode: a new technique for simultaneous isolation of several single units in the central nervous system from multiple unit records. J Neurosci Methods 8:391397. CrossRef Medline

McNaughton BL, Barnes CA, Gerrard JL, Gothard K, Jung MW, Knierim JJ, Kudrimoti H, Qin Y, Skaggs WE, Suster M, Weaver KL (1996) Deciphering the hippocampal polyglot: the hippocampus as a path integration system. J Exp Biol 199:173-185. Medline

McNaughton BL, Battaglia FP, Jensen O, Moser EI, Moser MB (2006) Path integration and the neural basis of the "cognitive map." Nat Rev Neurosci 7:663-678. CrossRef Medline

Mehta MR, Barnes CA, McNaughton BL (1997) Experience-dependent, asymmetric expansion of hippocampal place fields. Proc Natl Acad Sci U S A 94:8918-8921. CrossRef Medline

Moser EI, Kropff E, Moser MB (2008) Place cells, grid cells, and the brain's spatial representation system. Annu Rev Neurosci 31:69-89. CrossRef Medline

Muller RU, Kubie JL, Ranck JB Jr (1987) Spatial firing patterns of hip- pocampal complex-spike cells in a fixed environment. J Neurosci 7:19351950. Medline

Nitz DA (2006) Tracking route progression in the posterior parietal cortex. Neuron 49:747-756. CrossRef Medline

Nitz DA (2011) Path shape impacts the extent of CA1 pattern recurrence both within and across environments. J Neurophysiol 105:1815-1824. CrossRef Medline

O'Keefe J (1976) Place units in the hippocampus of the freely moving rat. Exp Neurol 51:78-109. CrossRef Medline

O'Keefe J, Dostrovsky J (1971) The hippocampus as a spatial map: preliminary evidence from unit activity in the freely-moving rat. Brain Res 34: 171-175. CrossRef Medline

O'Keefe J, Nadel L (1978) The hippocampus as a cognitive map. Oxford: Clarendon.

Quirk GJ, Muller RU, Kubie JL (1990) The firing of hippocampal place cells in the dark depends on the rat's recent experience. J Neurosci 10:20082017. Medline

Rivas J, Gaztelu JM, García-Austt E (1996) Changes in hippocampal cell discharge patterns and theta rhythm spectral properties as a function of walking velocity in the guinea pig. Exp Brain Res 108:113-118. Medline

Sargolini F, Fyhn M, Hafting T, McNaughton BL, Witter MP, Moser MB, Moser EI (2006) Conjunctive representation of position, direction, and velocity in entorhinal cortex. Science 312:758-762. CrossRef Medline

Singer AC, Karlsson MP, Nathe AR, Carr MF, Frank LM (2010) Experiencedependent development of coordinated hippocampal spatial activity representing the similarity of related locations. J Neurosci 30:11586-11604. CrossRef Medline

Skaggs WE, McNaughton BL (1998) Spatial firing properties of hippocampal CA1 populations in an environment containing two visually identical regions. J Neurosci 18:8455-8466. Medline

Sławińska U, Kasicki S (1998) The frequency of rat's hippocampal theta rhythm is related to the speed of locomotion. Brain Res 796:327-331. CrossRef Medline

Stackman RW, Taube JS (1998) Firing properties of rat lateral mammillary single units: head direction, head pitch, and angular head velocity. J Neurosci 18:9020-9037. Medline

Taube JS, Muller RU (1998) Comparisons of head direction cell activity in the postsubiculum and anterior thalamus of freely moving rats. Hippocampus 8:87-108. CrossRef Medline

Valerio S, Taube JS (2012) Path integration: how the head direction signal maintains and corrects spatial orientation. Nat Neurosci 15:1445-1453. CrossRef Medline

Wilent WB, Nitz DA (2007) Discrete place fields of hippocampal formation interneurons. J Neurophysiol 97:4152-4161. CrossRef Medline

Zhang SJ, Ye J, Miao C, Tsao A, Cerniauskas I, Ledergerber D, Moser MB, Moser EI (2013) Optogenetic dissection of entorhinal-hippocampal functional connectivity. Science 340:1232627. CrossRef Medline 